Letter

\title{
Diagnostic and prognostic value of microRNAs for cancers- strategies and approaches to improve the clinical utility
}

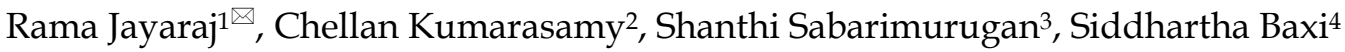 \\ 1. Clinical Sciences, College of Health and Human Sciences, Charles Darwin University, Ellengowan Drive, Casuarina, Northern Territory 0909, Australia \\ 2. University of Adelaide, North Terrace Campus, Adelaide, South Australia 5005, Australia. \\ 3. School of Biosciences and Technology, VIT University, Vellore, Tamil Nadu, India. \\ 4. Senior Clinical Oncologist, Genesis Cancer Care Centre, Bunbury, Western Australia 6230, Australia. \\ $\square$ Corresponding author: Senior Lecturer - Clinical Sciences, College of Health and Human Sciences, Charles Darwin University, Ellengowan Drive, Casuarina, \\ Northern Territory 0909, Australia. E-mail: Rama.Jayaraj@cdu.edu.au (RJ) \\ (C) Ivyspring International Publisher. This is an open access article distributed under the terms of the Creative Commons Attribution (CC BY-NC) license \\ (https://creativecommons.org/licenses/by-nc/4.0/). See http://ivyspring.com/terms for full terms and conditions.
}

Received: 2018.07.17; Accepted: 2018.09.14; Published: 2019.01.29

We have come across the paper titled, "Diagnostic and prognostic value of microRNA-628 for cancers", published by $\mathrm{Li}$ et al. in the Journal of Cancer [1]. The publication is of great interest to us, and we would like to put forth a few strategies and approaches in regards to the publication in the spirit of scientific inquiry.

Is miRNA ubiquitous in all forms of cancer? We would like to applaud $\mathrm{Li}$ and associates in their attempt to highlight miR-628 as both a promising diagnostic, as well as prognostic marker. However, the clinical utility of such a study is affected by the authors' decision to place the focus of the study upon miR-628. An unstated assumption made by the authors is that this study considers all forms of cancer to be identical to each other. It is known that different types of cancer have different physiological and genetic markers. Therefore the consideration that miR-628 is ubiquitous in all forms of cancer is a major misleading notion. This assumption further accentuated by the authors' decision not to perform any subgroup analysis based on 'cancer types', while simultaneously having a small set of published study data to conduct a meta-analysis.

Analysis of heterogeneity for diagnostic test accuracy studies: We also believe that the statistical analysis could benefit from the inclusion of the Tau $\left(\mathrm{T}^{2}\right)$-squared $\left(\mathrm{T}^{2}\right)$ statistic. Though the Chi-square and I-square statistical parameters are informative, they may be insufficient as they do not consider the threshold effect. Considering the between-study heterogeneity in study parameters, and the application of a random-effects model for the meta-analysis, Tau-square $\left(\mathrm{T}^{2}\right)$, as the parameter estimating variation or heterogeneity between the effects for test accuracy, may substantially improve the amount and clarity of the information obtained from Li et al.'s study [2]. Furthermore, we observed that the $Z$ value had not been estimated, is the test statistic for testing null hypothesis and used to derive the $P$ value, the addition of both of these statistical parameters is strongly suggested [3].

Statistical significance and effect size: Similarly, we would also like to highlight that statistical significance alone is not a sufficient parameter to judge the prognostic or diagnostic effectiveness of miR-628 in this study. Interpretation of effect size and its impact on the results of the study is crucial. Statistical significance as a resulting parameter is binary in its interpretation on the effect of any intervention and prognosis or diagnosis, whereas effect size allows for a much more nuanced interpretation of the observed results in a meta-analysis. Demonstration of effect size parameter, would, therefore, serve to improve the results presented in the study [4].

We understand that it may not be possible to retroactively add the suggestions we have proposed to the study already published by Li et al. However, it will most certainly benefit any future studies 
conducted in the similar field. We have written this letter in order to highlight strategies and propose improvements, such that, both the authors of this study and other researchers working in this field, could consider these points and publish higher-quality systematic reviews and meta-analysis of prognostic and diagnostic test accuracy studies, thereby benefiting the cycle of future scientific discovery towards clinical translation.

\section{Authors' contributions}

RJ conceived of this critical review and led the development of the letter to the editor. RJ and CK wrote the first draft of the letter, and coordinated and integrated comments from co-authors, SS, and SB critically revised and edited successive drafts of the manuscript. All authors read and approved the final version of the manuscript.

\section{Competing Interests}

The authors have declared that no competing interest exists.

\section{References}

1. Li J-H, Sun S-S, Fu C-J, Zhang A-Q, Wang C, Xu R, et al. Diagnostic and prognostic value of microRNA-628 for cancers. Journal of Cancer. 2018;9(9):1623.

2. Lee J, Kim KW, Choi SH, Huh J, Park SH. Systematic Review and Meta-Analysis of Studies Evaluating Diagnostic Test Accuracy: A Practical Review for Clinical Researchers-Part II. Statistical Methods of Meta-Analysis. Korean Journal of Radiology. 2015;16(6):1188-96. doi: 10.3348/kjr.2015.16.6.1188. PubMed PMID: PMC4644739.

3. Freemantle N, Geddes J. Understanding and interpreting systematic reviews and meta-analyses. Part 2: meta-analyses. Evidence Based Mental Health. 1998;1(4):102.

4. Coe R. It's the effect size, stupid: What effect size is and why it is important. 2002. 